\title{
EFFICIENT ATTRIBUTE REDUCTION ALGORITHM
}

\author{
Zhongzhi Shi, Shaohui Liu, Zheng Zheng \\ Institute Of Computing Technology,Chinese Academy of Sciences, Beijing, China
}

\begin{abstract}
Efficiency of algorithms is always an important issue concerned by so many researchers. Rough set theory is a valid tool to deal with imprecise problems. However, some of its algorithms' consuming time limits the applications of rough set. According to this, our paper analyzes the reasons of rough set algorithms' inefficiency by focusing on two important factors: indiscernible relation and positive region, and analyzes an equivalent and efficient method for computing positive region. And according to our research on the efficiency of some basic issues of rough set, a complete algorithm for the reduction of attributes is designed and its completeness is proved. Theoretical analysis and experimental results illustrate that our reduction algorithm is more efficient than some other algorithms.
\end{abstract}

Key words: Rough set, Positive region, Attribute Core, Attribute reduction algorithm, Discernibility matrix

\section{INTRODUCTION}

The classical rough set developed by Professor Pawlak is a valid mathematical theory, which has the ability to deal with imprecise, uncertain and vague information. In recent years, it has been applied in machine learning, data mining, knowledge acquiring successfully and has a great improvement of its theory and applications ${ }^{[2-7,20]}$.

At present, the research about rough set is mainly about mathematical properties, extended models, effective sub-algorithms and the relationship between rough set and other uncertain methods, etc. Rough set's effective algorithms include the algorithms about indiscernible classes, upper approximation, lower approximation, positive region, attribute reduction and 
attribute core, etc. In rough set, attribute reduction is one of the most important parts and there are several algorithms for it at present ${ }^{[8-17,20]}$. Hence, computation of attribute reduction is the key for rough set.

Attribute reduction is a process for reducing the redundant attributes, under keeping the classifying and decision ability of an information system. Skowron presented a discernibility matrix that is a useful tool for attribute reduction. Most of the disernibility matrix based algorithms first collect a decision table's discernibility information into a matrix, thus we can get the attribute core of an information system from the matrix easily. Because of the explicit presentation of discernibility information by discernibility matrix, it is simple for the computation of attribute core and attribute reduction sets, etc. Thus, many algorithms for computing attribute reduction are based on discernibility matrix. Unfortunately, there are also some problems about discernibility matrix, one of which is that the time complexity of computing discernibility matrix is so high that, when the data set is huge, it is time consuming to find the attribute reduction sets.

The inefficiency of attribute reduction algorithms limits the application of rough set. So, it is important to find efficient algorithms and algorithms for attribute reduction. For solving the problem, we first analyze the reason leading to these algorithms' high time complexity. Then, based on the concept of indiscernible relation and positive region, we research the properties of indiscernible relation and present a new method for computing positive region. Moreover, we present an incremental algorithm for computing positive region and at last, an attribute reduction algorithm is developed. We compare our attribute reduction algorithm with other attribute reduction algorithms and the results show that our algorithm is more efficient.

In section 2, we introduce some basic notions of rough set. In section 3, we analyze the properties of indiscernible relation and present an algorithm for computing positive region. In section 4, we discuss the incremental computation of positive region and design an efficient and complete attribute reduction algorithm. In section 5, we test our attribute reduction algorithm's validity and ability, and compare it with other attribute reduction algorithms. The last section is the conclusion and future work of this paper.

\section{BASIC NOTIONS OF ROUGH SET}

For the convenience of description, we introduce some basic notions of rough set at first.

Definition 1 A decision information system is defined as $S=\langle U, A, V, f\rangle$, where $U$ is a finite set of objects and $A=C \cup D$ is a finite set of attributes. $C$ is 
the condition attribute set and $D$ is the decision attribute set. With every attribute $a \square A$, set of its values $V_{a}$ is associated and $V=\cup_{a \in A} V_{a}$. Each attribute has a determine function $f: U \times A \rightarrow V$.

Definition 2 Given an information system $S$ and an attribute subset $\boldsymbol{B} \subseteq$ $A$, we can define an indiscernible relation by the following:

$\operatorname{IND}(B)=\{(x, y) \in U \times U \square \forall b \in B, b(x)=b(y)\}$.

It is obvious that $\operatorname{IND}(B)$ is an indiscernible relation and an object $x$ 's indiscernible class $[x]_{I N D(B)}$ in $B$ can be defined as follows

$[x]_{I N D(B)}=\{y: y \in U, y I N D(B) x\}$.

For the convenience of description, we use $B$ to replace $\operatorname{IND}(B)$ where there isn't any confusion.

Definition 3 Given a partition $R$, and a subset $X \subseteq U$, we can define a $B$ lower approximation of $\mathrm{X}$ in $\mathrm{U}$ and a $B$-upper approximation of $X$ in $U$ by the following:

$$
\begin{array}{r}
\underline{B} X=\left\{x \in U:[x]_{B} \subseteq X\right\}, \\
\bar{B} X=\left\{x \in U:[x]_{B} \cap X \neq \phi\right\} .
\end{array}
$$

Definition $4 P O S_{P}(Q)=\bigcup \underline{P} X$ is the $P$ positive region of $Q$, where $P$ and $Q$ are both attribute sets $\boldsymbol{E F}^{\prime} a n$ information system and $\underline{P} X$ is the $P$ lower approximation of an object set $X$.

Definition 5 An attribute $r$ of an information system is said to be relatively dispensable or superfluous if $P O S_{P}(Q)=P O S_{(P-\{r\})}(Q)$, otherwise, relatively indispensable. A relative reduction of $P$ is a set of attributes $S \subseteq P$ such that all attributes $a \square P-S$ are relatively dispensable, all attributes $a \square S$ are relatively indispensable and $P O S_{S}(Q)=P O S_{P}(Q)$. We use term $\operatorname{Red}_{D}(B)$ to denote the family of relative reducts of $B$. $\operatorname{Core}_{D}(B)=\cap \operatorname{Re} d_{D}(B)$ is called the D-core of attribute set B.

\section{BASIC ALGORITHMS OF ROUGH SET}

\subsection{Indiscernible Relation}

Indiscernible relation is one of the most important concepts in rough set. It is the basis to define some other basic concepts, such as upper approximation and lower approximation, etc. Consequently, the complexity of computing indiscernible relation influences other algorithms' executing time directly.

The general method of computing indiscernible relation $\operatorname{IND}(B)$ is that: compare each object in $U$ with all of the other objects one by one. And the objects with the same values of attributes in $B$ belong to the same indiscernible class and the objects with different values of attributes in $B$ 
belong to different indiscernible classes. At the worst case, the method need to compare two objects with $|\mathbf{U}|^{2}$ times, i.e., the time complexity of the method is $\mathbf{O}\left(|\mathbf{B} \| \mathbf{U}|^{2}\right)^{[2,17]}$.

Based on the definition of $\operatorname{IND}(B)$, indiscernible class has the following property:

Property 1: The two objects belong to the same indiscernible class, iff they have the same values of the attributes in $B$.

Based on this property, we can improve the general algorithm for computing $\operatorname{IND}(B)$. Before calculating the indiscernible relation, we list the objects in $U$ according to the values of attributes in $B$ first. The algorithm is as follows:

Algorithm1: Algorithm for computing all indiscernible classes of $\operatorname{IND}(B)$

Input: $S=<U, A, V, f>, U=\left\{u_{1}, u_{1}, \ldots, u_{\mid \varphi}\right\}$, and $B \subseteq A$;

Output: $U / B$.

Step 1 Arrange the objects in $U$ based on the values of attributes in $B$;

Step $2 s=1, j=1, B_{l}=\left\{u_{l}\right\}$;

Step 3 for $i=2$ to $\mid \boldsymbol{U}$ do

if $\boldsymbol{u}_{\mathrm{i}}$ and $\boldsymbol{u}_{\mathrm{j}}$ have the same values of attributes in $B$,

then $B_{s}=B_{s} \cup\left\{\mathbf{u}_{i}\right\}$;

$$
\text { else } s=s+1, B_{s}=\left\{\mathbf{u}_{i}\right\}, j=i \text {. }
$$

The time complexity of ordering the objects in $U$ is $O(|B \| U \log | U)$. In step 3, each object in $U$ needs to be searched only once, so the complexity of step 3 is $O(|B \| U|)$. Therefore, the complexity of algorithm 1 is $O(|\mathrm{~B} \| U| \log |U|)$, which is lower than the general algorithm.

If $\operatorname{IND}(B)$ is known, we can calculate the $B$-lower approximation or $B$ upper approximation of object set $X \subseteq U$ only by searching whether the $B$ indiscernible classes are included in $X$ or have intersections with $X$. The number of $B$ indiscernible classes is $\mid U$ at the worst case. Thus, when the $B$ indiscernible classes are known, the time complexities of lower and upper approximations are both $O\left(U_{U}\right)$.

Given an information system $S$, supposing $B \subseteq A$ and $X \subseteq U$, we can deduce from the time complexity of algorithm 1 that the time complexities of calculating $X$ 's lower approximation, $X$ 's upper approximation, the attribute core of $B$ and $\operatorname{Red}_{\mathrm{D}}(B)$ are $\mathrm{O}(|A||U| \log |U|), \mathrm{O}(|A||U| \log |U|), \mathrm{O}\left(|A|^{2}|U| \log \mid U\right)$ and $\mathrm{O}\left(\left(2^{|A|}|A \| U \log | U\right)\right.$ respectively. They are lower than the corresponding algorithms' complexities in $[16,20]$, which are $\mathrm{O}\left(|A| \|\left.\right|^{2} \mid\right), \mathrm{O}\left(|A||U|^{2} \mid\right)$, $\mathrm{O}\left(|A|^{2}|U|^{2} \mid\right)$ and $\mathrm{O}\left(\left(2^{|A|}|A \| U|^{2}\right)\right.$. 


\subsection{Positive Region}

Because the definitions of attribute core and relative attribute reduction are based on positive region, computation of positive region is the key of rough set.

According to the definition of $\operatorname{POS}_{P}(Q)$, the general method of computing $P O S_{P}(Q)$ is that: first, compute the indiscernible classes based on $Q$, and suppose the result is $U / Q=\left\{Q_{1}, Q_{2}, \ldots Q_{n}\right\}, 0<n \leq|U|$; then, calculate $\underline{P}\left(Q_{i}\right)$ respectively $(i=1,2, \ldots, \mathrm{n})$. When $U / P$ and $U / Q$ are known, the time complexity of computing $P\left(Q_{i}\right)$ is $O(|U|)$, so the time complexity of computing $P O S_{P}(Q)$ is $O\left(\mid U^{2}\right)^{[16,20]}$.

Based on the definition of positive definition, we can develop a new algorithm for computing positive region. We introduce a theorem as follows at first.

$$
\text { Theorem 1: } \operatorname{POS}_{P}(Q)=\bigcup_{Y \in U / P \text { and }|Y / Q|=1}^{\bigcup}
$$

Proof.

Suppose $U / P=\left\{P_{1}, P_{2,} \ldots P_{m}\right\}(0<m \leq|U|), U / Q=\left\{Q_{1}, Q_{2}, \ldots Q_{n}\right\}(0<n \leq \mid U)$ and $x \in P O S_{P}(Q)$.

Because

$$
\operatorname{POS}_{P}(Q)=\bigcup_{X \in U / Q}(X)=\underline{P}\left(Q_{1}\right) \cup \underline{P}\left(Q_{2}\right) \cup \ldots \underline{P}\left(Q_{n}\right),
$$

there is an indiscernible class $Q_{k} \in U / Q(1 \leq k \leq n)$ that satisfies $x \in P Q_{k}$. In similar way, because $\underline{P Q_{k}}=\bigcup_{P_{1} \leq Q_{k}} P_{i}$, there is an indiscernible class $\mathbf{P}_{\mathbf{l}} \in \mathbf{U} / \mathbf{P}(1 \leq \mathbf{l} \leq \mathbf{m})$ that satisfies $\mathbf{x} \in \mathbf{P}_{\mathbf{l}}$. Because $\mathbf{P}_{1} \subseteq \mathbf{Q}_{k}$, for all objects that belong to $\mathbf{P}_{\mathbf{l}}$, they not only have the same values of attributes in $\mathrm{P}$, but also have the same values of attributes in $Q$. So $P / Q=\left\{P_{1}\right\}$, that is $\left|P_{1} / Q\right|=1$. And

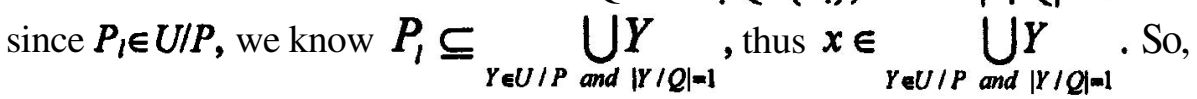

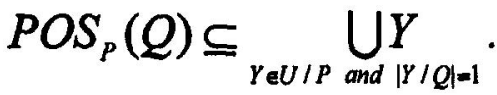

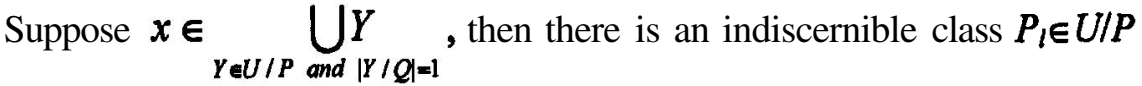
$(1 \leq l \leq m)$ that satisfies $\left|P_{l} / Q\right|=1$ and $x \in P_{1}$. And since $\left|P_{l} / Q\right|=1$, thus $P_{l} / Q=\left\{P_{l}\right\}$, that is, for all objects in $\boldsymbol{P}_{\boldsymbol{l}}$, they have the same values of the attributes in $Q$. And because $P \leq U$, there is an indiscernible class $Q_{k} \in U / Q(1 \leq k \leq n)$ such that $P \subseteq Q_{k}$. According to the definition of lower approximation, we know $\underline{P} P_{l} \subseteq P Q_{k}$, and since $\underline{P}_{l}=P_{l}$, thus $P \subseteq P Q_{k} \subseteq P O S_{P}(Q)$, so $x \in P O S_{P}(Q)$. Therefore, $\bigcup_{Y \in U / P} Y \subseteq P_{P} S_{P}(Q)$.

Based on all above, theorem 1 holds. 
According to theorem 1, we develop a new algorithm for computing positive region.

Algorithm 2: Improved algorithm for computing positive region

Input: $S=<U, A, V, f>$, and $P, Q \subseteq A$;

Output: $\operatorname{POS}_{P}(Q)$.

Step 1: Compute the indiscernible classes based on $P$ and suppose the result is $\left\{P_{1}, P_{2}, \ldots P_{m}\right\}, 0<m \leq|U|$;

Step 2: $\operatorname{POS}_{p}(Q)=\phi$;

Step 3: For $i=1$ to $m$ do

Computing the $Q$ indiscernible classes of $\boldsymbol{P}_{\boldsymbol{1}}$. And if there is only one resulting indiscernible class, then $\operatorname{POS}_{p}(Q)=P O S_{p}(Q) \cup P_{i}$.

The time complexity of step 1 is $O(|P \| U| \log |U|)$. The time complexity of the $i$ th loop in step 3 is $\mathrm{O}\left(\left|Q \| P_{i}\right| \log \left|P_{i}\right|\right)$, so the time complexity of step 3 is $O\left(\left|Q \| P_{1}\right| \log \left|P_{1}\right|\right)+\mathrm{O}\left(\left|Q \| P_{2}\right| \log \left|P_{2}\right|\right)+\ldots+\mathrm{O}\left(\left|Q \| P_{m}\right| \log \left|P_{m}\right|\right)$. Since $\left|P_{1}\right| \leq|U|$ and $\left|P_{1}\right|+\left|P_{2}\right|+\ldots+\left|P_{m}\right|=|U|$, we have $O\left(\left|Q \| P_{l}\right| \log \left|P_{l}\right|\right)+\mathrm{O}\left(\left|Q \| P_{2}\right| \log \left|P_{2}\right|\right)+\ldots+$ $\mathrm{O}\left(\left|Q \| P_{m}\right| \log \left|P_{m}\right|\right) \leq \mathrm{O}(|Q \| U| \log |U|) \mid$.

Because $|Q| \leq|A|$ and $|P| \leq|A|$, the time complexity of computing positive region is reduced to $\mathrm{O}(|A||U| \log |U|)$ using algorithm 2. We can deduce from above analysis that the complexity of computing attribute core, and attribute reduction is decreased to $\mathrm{O}\left(|A|^{2}|U| \log \mid U\right), \mathrm{O}\left(\left(2^{|K|}|A|^{2}|| U|\log | U \mid\right)\right.$.

\section{EFFICIENT ALGORITHMS FOR ATTRIBUTE REDUCTION}

Since computing attribute reduction sets is a NP hard problem, we often use heuristic methods to find the optimal or hypo-optimal attribute reduction sets. One of the common features of these methods is that they use the importance degree of attributes as the heuristic information. By taking different importance degrees of attributes, there exist different kinds of attribute reduction algorithms, such as the positive region based attribute reduction algorithm ${ }^{[9,10,16]}$, the discernible matrix and attribute frequency based attribute reduction algorithm ${ }^{[12,15]}$, and information entropy based attribute reduction algorithm ${ }^{[14]}$, etc.

Unfortunately, most of the algorithms ${ }^{[9,10,12,14]}$ are not complete. As a result, it is not assured to acquire the attribute reduction set. For example, Table 1 is a decision table, $\{a, b, c, d, e\}$ is the condition attribute set and $\{\mathrm{f}\}$ is the decision attribute set. Using the algorithms ${ }^{[9,10,12,14]}$ we mentioned before, the resulting condition attribute reduction set is $\{a, b, c\}$. However, $a$ is an attribute that can be ignored and $\{b, c\}$ is the result in deed. 
Table1 A Decision System DS

\begin{tabular}{|c|c|c|c|c|c|c|}
\hline U & a & b & c & d & e & f \\
\hline 1 & 1 & 1 & & 1 & 1 & 1 \\
\hline & 2 & 2 & 1 & 1 & 1 & 2 \\
\hline 3 & 3 & 3 & 3 & 3 & 3 & 3 \\
\hline 4 & 4 & 4 & 3 & 3 & 3 & 4 \\
\hline 5 & 5 & 5 & 5 & 5 & 5 & 5 \\
\hline 6 & 6 & 6 & 5 & 5 & 5 & 6 \\
\hline 7 & 7 & 7 & 7 & 7 & 7 & 7 \\
\hline 8 & 8 & 8 & 7 & 7 & 7 & 8 \\
\hline 9 & 9 & 9 & 9 & 9 & 9 & 9 \\
\hline 10 & 10 & 10 & 9 & 9 & 9 & 10 \\
\hline 11 & 11 & 11 & 11 & 11 & 11 & 11 \\
\hline 12 & 12 & 11 & 12 & 11 & 11 & 12 \\
\hline
\end{tabular}

\begin{tabular}{|l|l|l|l|l|l|l|}
\hline $\mathrm{U}$ & $\mathrm{a}$ & $\mathrm{b}$ & $\mathrm{c}$ & $\mathrm{d}$ & $\mathrm{e}$ & $\mathrm{f}$ \\
\hline 13 & 13 & 13 & 13 & 13 & 13 & 13 \\
\hline 14 & 14 & 13 & 14 & 13 & 13 & 14 \\
\hline 15 & 15 & 15 & 15 & 15 & 15 & 15 \\
\hline 16 & 16 & 15 & 16 & 15 & 15 & 16 \\
\hline 17 & 17 & 17 & 17 & 17 & 17 & 17 \\
\hline 18 & 18 & 17 & 18 & 17 & 17 & 18 \\
\hline 19 & 19 & 19 & 19 & 19 & 19 & 19 \\
\hline 20 & 20 & 19 & 20 & 19 & 19 & 20 \\
\hline 21 & 21 & 21 & 21 & 21 & 21 & 21 \\
\hline 22 & 22 & 21 & 22 & 21 & 21 & 22 \\
\hline 23 & 23 & 23 & 23 & 23 & 23 & 23 \\
\hline 24 & 23 & 24 & 24 & 23 & 23 & 24 \\
\hline
\end{tabular}

\begin{tabular}{|l|l|l|l|l|l|l|}
\hline $\mathrm{U}$ & $\mathrm{a}$ & $\mathrm{b}$ & $\mathrm{c}$ & $\mathrm{d}$ & $\mathrm{e}$ & $\mathrm{f}$ \\
\hline 25 & 25 & 25 & 25 & 25 & 25 & 25 \\
\hline 26 & 25 & 26 & 26 & 25 & 25 & 26 \\
\hline 27 & 27 & 27 & 27 & 27 & 27 & 27 \\
\hline 28 & 27 & 28 & 27 & 28 & 27 & 28 \\
\hline 29 & 29 & 29 & 29 & 29 & 29 & 29 \\
\hline 30 & 29 & 30 & 29 & 30 & 29 & 30 \\
\hline 31 & 31 & 31 & 31 & 31 & 31 & 31 \\
\hline 32 & 31 & 32 & 31 & 32 & 31 & 32 \\
\hline 33 & 33 & 33 & 33 & 33 & 33 & 33 \\
\hline 34 & 33 & 33 & 34 & 34 & 33 & 34 \\
\hline 35 & 35 & 35 & 35 & 35 & 35 & 35 \\
\hline 36 & 35 & 35 & 36 & 35 & 36 & 36 \\
\hline
\end{tabular}

Besides, The time complexities of the algorithms ${ }^{[9,10,12,14]}$ are all $O\left(|A|^{2}|U|^{2}\right)$. Reference 15 presented an attribute reduction algorithm based on attribute sequence and discernible matrix. Its time complexity is also $O\left(|\mathbf{A}|^{2}|U|^{2}\right)$ and its spatial complexity is $O\left(|A||U|^{2}\right)$; Reference 16 presented an attribute reduction algorithm, but having a relatively high time complexity, $O\left(\left.|\mathrm{~A}|\right|^{3} U^{2}\right)$.

\subsection{The Incremental Computation Of Positive Region}

How to calculate the attributes' importance degree is the key problem in heuristic attribute reduction algorithms. Also, it is important for improving the efficiency of algorithms. In the remainder of our paper, we mainly discuss the algorithms for the condition attribute reduction of a decision system $S=<U, C \cup D, V, \beta$. In these algorithms, the importance degree of an attribute $a \in C-R \square R \subset C \square$ to the decision attribute set $D, S G F(a, R, D)$, is defined as follows:

$S G F(a, R, D)=\gamma_{R \cup\{a\}}-\gamma_{R}$, where $\left.\gamma_{R}=\mid P O S_{R}(D)\right)|| \mid U$.

From the formula, we know positive region is the key for computing $S G F(a, R, D)$. According to algorithm 2, the time complexity of computing $\gamma_{R(a)}$ is $O((|R|+|D|+1)|U| \log |U|)$.

Based on the definition of indiscernible relation, we can prove the following property easily.

Property 2: Suppose $S=<U, C \cup D, V, \beta$ is a decision system, $R \subset C$, $a \in C-R$, and $U / R=\left\{R_{1}, R_{2}, \ldots, R_{m}\right\} \quad 0<m \leq|U|$, thus, $U /(R \cup\{a\})=R_{1} /\{a\} \cup$ $R_{2} /\{a\} \cup \ldots \cup R_{\mathrm{m}} /\{a\}$, that is $U /(R \cup\{a\})=\bigcup_{X \in U / R} X /\{a\}$.

According to theorem 1 and property 2, it is easy to conclude that:

Corollary 1: 


$$
P O S_{R \cup(a)}(Q)=\underset{Y \in U /(R \cup(a)) \text { and }|Y / Q|=1}{\cup} Y=\{\cup Y: Y \in \underset{x \in U / R}{\bigcup} X /\{a\},
$$
and $|Y / Q|=1\}$.

Based on corollary 1, we present an incremental algorithm for computing positive region as follows:

Algorithm 3: Incremental algorithm for computing positive region Input: $S=<U, C \cup D, V, \beta\rangle, U / R=\left\{R_{1}, R_{2}, \ldots R_{m}\right\}, 0<m \leq|U|$;

Output: $P O S_{R \cup\{a\}}(D)$.

Step 1: Calculate the indiscernible classes $U / R \cup\{a\}$ according to $U / R$ and suppose the result is $W_{1}, W_{2}, \ldots W_{n}, 0<n \leq|U|$;

Step 2: $\operatorname{POS}_{R \cup(a)}(D)=\phi$;

Step 3: For $i=1$ to $m$ do

Classify $W_{l}$ based on $D$ and if the resulting indiscernible relation has only one indiscernible class, then $\operatorname{POS}_{R \cup(a)}(D)=P O S_{R \cup(a)}(D) \cup W_{1}$.

Similar with the analysis of algorithm 2's complexity, when U/R is known, we can deduce that the time complexity of algorithm 3 is $\mathrm{O}((|D|+1)|U| \log |U|)$. It is obviously that the complexity is irrelevant with $|R|$. Therefore, when we incrementally compute positive region $\operatorname{POS}_{R \cup(a)}(D)$, we can save the result of $U / R$ first to reduce the complexity.

In the similar way, when $U / R$ and $\operatorname{POS}_{R}(D)$ are known, the complexity of computing $S G F(a, R, D)$ is $\mathrm{O}((|D|+1)|U| \log \mid U)$.

\subsection{A New Attribute Reduction Algorithm}

Based on the above understanding, we develop an efficient attribute reduction algorithm.

Algorithm 4: A new attribute reduction algorithm Input: $S=\langle U, C \cup D, V, f>$;

Output: the D-attribute reduction set of C, i.e., $R E D$.

Step 1: Compute the $D$-attribute core of $C, \operatorname{CORE}_{D}(C)$;

Step 2: $R E D=\operatorname{CORE}_{D}(C)$;

Step 3: Compute $P O S_{C}(D), U / R E D$ and $P O S_{R E D}(D)$;

Step 4: While $\left|P O S_{C}(D)\right| \neq\left|P O S_{R E D}(D)\right|$, do

1) Find the attribute $a$ that the value of $S G F(a, R E D, D)$ is maximal;

2) $R E D=R E D \cup\{a\}$ and add $a$ to the tail of RED;

3) Use algorithm 3 to compute $U / R E D$ and $P O S_{R E D}(D)$;

Step 5: Get the last attribute $a$ from $R E D$ and if $a \in C O R E_{D}(C)$,

then $R E D$ is the result and the algorithm is end

else if $\left|P O S_{C}(D)\right|=\left|\operatorname{POS}_{R E D-\{a\}}(D)\right|$

$R E D=R E D \square\{a\}$; go to step 5 . 
Relative attribute core is the start point of algorithm 4 and with step 4 it is assured to find a subset $R E D$ of $C$ that satisfies $\left|P O S_{C}(D)\right|=\left|P O S_{R E D}(D)\right|$. The condition attributes are arranged in $R E D$ with their importance degrees. The attributes arranged in the frontal part of $R E D$ are core attributes, and the less importance degree of the attributes, the latter positions the attributes locate. In step 5, if some attributes are ignorable, the attributes with less importance degree are reduced first. At last, the attributes remained in $R E D$ are all that can't be deleted. Therefore, the resulting RED is the attribute reduction set we want. Based on the above, we can conclude that our algorithm is complete for finding condition attribute reduction set.

\subsection{Analysis Of Algorithm 4's Complexity}

The time complexity of step 1 is $O\left((|C|+|\mathrm{D}|)^{2}|U \log | U \mid\right)$ and the time complexity of step 3 is $O((|C|+|\mathrm{D}|)|U| \log |U|)$. In each loop of step 4, the first step need to compute $S G F(a, R E D, D)$ with $|C-R E D|$ times, and the complexity of the third step is $\mathrm{O}((|D|+1)|U \log | U \mid)$. In the worst case, it need $|C|$ times to execute step 4 , that is, in total, it need to compute the importance degree of attributes $|C|+(|C|-1)+\ldots+1=|C|(|C|+1) / 2$ times. Since the time complexity of computing $S G F(a, R, D)$ is $\mathrm{O}((|D| \square 1)|U| \log |U|)$, the time complexity of step 4 is $\mathbf{O}\left(|C|^{2}(|D|+1)|U| \log \mid U\right)$.

Using the method mentioned in [24], any decision table can be transformed to a decision table with only one decision attribute, so we might as well suppose $\mathrm{D}=\{\mathrm{d}\}$, i.e., $|D|=1$, and according to the above analysis the time complexity of algorithm 4 is $\mathbf{O}\left(|C|^{2}|U| \log |U|\right)$.

Based on the above analysis, we can know the spatial complexity of algorithm 4 is $O(|C||\mathrm{U}|)$.

\section{RESULTS OF EXPERIMENT}

Table 2 Comparison of The Three Attribute Reduction Algorithms

\begin{tabular}{|l|l|l|l|l|l|l|l|l|}
\hline \multirow{2}{*}{ Decision table } & \multirow{2}{*}{$n$} & \multirow{2}{*}{$m$} & \multicolumn{2}{|l|}{$\mathrm{A}_{1}$} & \multicolumn{2}{l|}{$\mathrm{A}_{2}$} & \multicolumn{2}{l|}{$\mathrm{A}_{3}$} \\
\cline { 5 - 11 } & & & $m_{1}^{\prime}$ & $t_{l}(\mathrm{sec})$ & $m_{2}^{\prime}$ & $t_{2}(\mathrm{sec})$ & $m_{3}^{\prime}$ & $t_{3}(\mathrm{sec})$ \\
\hline Table 1 & 36 & 5 & 3 & 0.001 & 2 & 0.01 & 2 & 0.001 \\
\hline BUPA Liver Disorders & 345 & 6 & 3 & 0.06 & 3 & 0.12 & 3 & 0.02 \\
\hline $\begin{array}{l}\text { Voting Records } \\
\text { Database }\end{array}$ & 435 & 16 & 9 & 0.16 & 10 & 0.94 & 9 & 0.05 \\
\hline $\begin{array}{l}\text { Tic-Tac-Toe Endgame } \\
\text { database }\end{array}$ & 958 & 9 & 8 & 0.86 & 8 & 0.93 & 8 & 0.17 \\
\hline Chess End-Game & 3196 & 36 & 29 & 789.5 & 29 & 265.1 & 29 & 0.52 \\
\hline Mushroom Database & 8154 & 22 & 4 & 7603.6 & 5 & 470.8 & 5 & 3.92 \\
\hline
\end{tabular}


In order to test the validity and ability of algorithm 4, we use the data of table 1 and also collect some data sets from UCI database, then we program based on our algorithm. Our experiment is carried on the computer whose frequency is PIII 800, EMS memory is $128 \mathrm{M}$, and operating system is WIN2000. We compare the algorithms in reference [12] and [16] with our algorithm. $\mathbf{A}_{\mathbf{1}}$ is the algorithm in [12], $\mathbf{A}_{\mathbf{2}}$ is the algorithm in reference [16] and $\mathbf{A}_{\mathbf{3}}$ is ours (algorithm 4). We suppose $n$ is the number of the objects, $m$ is the number of condition attributes before reduced, $m_{i}^{\prime}$ is the number of condition attributes after reduced using $\mathbf{A}_{i}$ and $\boldsymbol{t}_{i}$ is the executing time of $\mathbf{A}_{i}$. The results are shown in table 2 .

From table 2, it is obvious that $\mathbf{A}_{\mathbf{3}}$ is more efficient than $\mathbf{A}_{\mathbf{1}}$ and $\mathbf{A}_{\mathbf{2}}$. With the increasing of objects' number, the improving of $\mathbf{A}_{\mathbf{3}}$ 's efficiency is greater. In the same case, the efficiency of $A_{1}$ decreases due to its high spatial complexity, $O\left(|A||U|^{2}\right)$. The results of experiments are consistent with our analysis. It proves our algorithm's validity and efficiency, and shows that our algorithm is more suitable to huge data set.

\section{CONCLUSION AND FUTURE WORK}

In rough set, fuzzy problems are discussed by upper and lower approximation and we can use some relevant mathematical formula to get results. Rough set is objective and based on original data set totally. Nowadays, because of rough set's unique feature, more and more scholars devoted into the research on it and made a great success. However, a lot of problems are needed to be resolved. Now, there are so little applications of rough set theory that have obvious benefits in industry ${ }^{[5]}$, so it is important to find faster and more efficient algorithms to advance it.

In this paper, the properties of indiscernible relation are discussed and a new algorithm computing positive region is presented and proved. Based on these, we analyze the incrementally computing of positive region. Then, we develop an attribute reduction algorithm, which is complete and efficient with time complexity $\mathbf{O}\left(|C|^{2}|\mathcal{U} \log | U \mid\right)$. Our simulation results show that our algorithm is better than many existed attribute reduction algorithms. In addition, due to the efficiency of our algorithm, it is useful to the application of rough set and helpful to apply it in dealing with huge data set. The future work of this paper includes feature selecting with rough set, constructing a better classifier that is suitable to decision support and using other uncertain methods to improve the classifying ability. 


\section{ACKNOWLEDGMENT}

This work is supported by the National Science Foundation of China (No.6007319, 60173017, 90104021) and the Nature Science Foundation of Beijing (No. 4011003).

\section{REFERENCE}

1. Z. Pawlak. Rough Sets, International Journal of Computer and Information Science, 1982, 11(5): 341 356

2. Z. Pawlak. Rough Sets: Theoretical Aspects of Reasoning about Data, Dordrecht: Kluwer Academic Publishers, 1991

3. A. Skowron. Rough Sets and Boolean Reasoning, In: W. Pedrycz ed. Granular Computing: An emerging Paradigm, New York : Physica-Verlag, 2001

4. Q. Liu, Rough Set and Rough Reasoning, Scientific Press, 2001

5. W. Ziako. Rough Sets: Trends, Challenges, and Prospects, In: W. Ziarko, Y. Y. Yao ed. Rough Sets and Current Trends in Computing(RSCTC 2000), Berlin: Springer-verlag, 2001

6. Q.Liu, S.H. Huang, L.W Yao, Rough Set Theory: Present State And Prospects, Computer Science, 1997, 24(4): 1 5

7. J. Wang, D.Q.Miao, Rough Set Thoery and Its Application: A Survey, Pattern Recognition And Artificial Intelligence, 1996, 9(4): 337 344

8. A. Skowron, C. Rauszer. The Discernibility Matrics and Functions in Inforamtion System. In: R.Slowinski edDIntelligent Decision Support Handbook of Applications and Advances of the Rough Sets Theory. Dordrecht: Kluwer Academic Publishers, 1992, 331 362

9. X.H. Hu, N. Cercone, Learning in Relational Databases: A Rough Set Approach., International Journal of Computational Intelligence, 1995, 11(2): 323 338

10. J. Jelonek, K. Krawiec, R. Slowinski. Rough Set Reduction of Attributes and Their Domains for Neural Networks, International Journal of Computational Intelligence, 1995, 11(2): 339 347

11. Q.Liu, S.H. Liu, F. Zheng, Rough Logic And Its Applications, Journal of Software, 2001, 12(3): $\mathbf{4 1 5 \square 4 1 9}$

12.J. Wang, R. Wang, D.Q. Miao, Data Enriching Based on Rough Set Theory, Chinese Journal of Software, 1998, 21(5): 393 399

13.D. Pan, Q.L. Zheng, An Adaptive Searching Opima: Algorithm for The Attribute Reducts, Journal of Computer Research \& Development, 2001, 38(8) $0904-910$

14.D.Q. Miao, G.R. Hu,A Heuristic Algorithm for Reduction of Knowledge, Journal of Computer Research \& Development, 1999, 36(6): 681 684

15.J. Wang, J. Wang. Reduction Algorithms Based on Discernbility Matrix: The Ordered Attributes Method, Journal of Computer Science \& Technology, 2001, 16(6): 489 504

16.J.W. Guan, D.A. Bell. Rough Computational Methods for Information Systems, Artificial Intelligences, 105(1998): 77 103

17.L.Y. Chang, G.Y. Wang, Y. Wu, An Approach for Attribute Reduction and Rule Generation Based on Rough Set Theory, Journal of Software, 1999, 10(11): 1206 1211

18.N. Zhong, A. Skowron, S. Ohsuga (Eds.) New Directions in Rough Sets, Data Mining, and Granular-Soft Computing(RSFDGrC'99), Berlin: Springer-Verlag, 1999 
19.E. Orlowska. (Ed.) Incomplete Information: Rough Set Analysis, New York : PhysicaVerlag, 1998

20. W.X. Zhang, W.Z. Wu, J.Y. Liang, D.Y. Li, Rough Set Theory And Methods, Scientific Press, 2001

21.J. Wroblewski, Finding Minimal Reducts Using Genetic Algorithm, ICS Research report 16/95, Warsaw University of Technology

22.W. Ziarko, The Discovery, Analysis, and Representation of Data Dependencies In Database, in G. Piatesky-Shapiro, W. Frawley (eds), Proceedings of the IJCAI Workshop On Knowledge Discovery, AAAI Press, 1991, 195 212

23.S.K.M. Wong, W. Ziarko, On Optimal Decision Rules in Decision Tables, Bulletin, Polish Academy of Sciences, 1985, 33(11-12): 693 696

24.G.Y. Wang, Rough Set Theory and Knowledge Acquisition, Press of Xi'an Jiaotong University, 2001 